\title{
Inspecting rapidly moving surfaces for small defects using CNN cameras
}

\author{
Andreas Blug*, Daniel Carl, Heinrich Höfler \\ Fraunhofer-Institute for Physical Measurement Techniques IPM, \\ Heidenhofstr. 8, 79110 Freiburg, Germany
}

\begin{abstract}
A continuous increase in production speed and manufacturing precision raises a demand for the automated detection of small image features on rapidly moving surfaces. An example are wire drawing processes where kilometers of cylindrical metal surfaces moving with $10 \mathrm{~m} / \mathrm{s}$ have to be inspected for defects such as scratches, dents, grooves, or chatter marks with a lateral size of $100 \mu \mathrm{m}$ in real time. Up to now, complex eddy current systems are used for quality control instead of line cameras, because the ratio between lateral feature size and surface speed is limited by the data transport between camera and computer. This bottleneck is avoided by "cellular neural network" (CNN) cameras which enable image processing directly on the camera chip. This article reports results achieved with a demonstrator based on this novel analogue camera - computer system. The results show that computational speed and accuracy of the analogue computer system are sufficient to detect and discriminate the different types of defects. Area images with $176 \mathrm{x} 144$ pixels are acquired and evaluated in real time with frame rates of 4 to $10 \mathrm{kHz}$ - depending on the number of defects to be detected. These frame rates correspond to equivalent line rates on line cameras between 360 and $880 \mathrm{kHz}$, a number far beyond the available features. Using the relation between lateral feature size and surface speed as a figure of merit, the CNN based system outperforms conventional image processing systems by an order of magnitude.
\end{abstract}

Keywords: Quality control, image processing, surface inspection, wire drawing, cellular neural networks

\section{INTRODUCTION}

Within industrial manufacturing, imaging and image processing systems are often crucial elements to the production process especially in automation and quality control. Therefore, they have a great leverage effect on other industries [1]. To maintain or even increase this market share, they have to cope with the increasing production speed and precision. This makes it important to overcome their limitations like the temporal resolution given by the frame rate and the latency time. A typical application in quality control are wire drawing processes, because they work on high feeding rates in the range of $10 \mathrm{~m} / \mathrm{s}$ and the quality of the resulting product is defined by the quality of the surface when the wire passes a die. Even rather small surface flaws with a lateral size of $100 \mu \mathrm{m}$ are able to cause breakage of the wire [2-4]. According to the sampling theorem, the theoretical limit for the minimum line frequency is $200 \mathrm{kHz}$. However, for a robust detection three lines per defect or a line frequency of $300 \mathrm{kHz}$ is desirable - which is not feasible for conventional image processing systems whose maximum frame rates are $140 \mathrm{kHz}$ or below $[5,6]$. The fastest wire drawing inspection system available on the market based on line cameras reaches just $35 \mathrm{kHz}$ [7]. Therefore, the majority of quality assurance systems for wire drawing processes today are based on eddy-current principles [8, 9]. The problem with these systems is that they require a complex mechanical setup in which point sensors rotate around the wire and that it is almost impossible to find false detections in the signals from kilometers of wire. Images are far easier to interpret, even if the exact position is unknown.

To overcome this situation, we used cameras based on cellular neural networks (CNN) which enable the integration of a "single instruction, multiple data" (SIMD) processor architecture into the electronic circuitry of CMOS camera pixels. Compared to conventional image processing, this novel camera technique offers two major advantages: Firstly, image preprocessing on the camera chip reduces data transfer between camera and processor which forms a common bottleneck in conventional image processing systems. Secondly, SIMD processors are in particular efficient in terms of computational speed and power consumption for low level image processing operations [10]. This paper discusses the properties of $\mathrm{CNN}$-cameras for surface inspection applications at the example of the wire drawing application.

*Andreas.blug@ipm.fraunhofer.de; Phone: +49 761 8857-328; Fax: +49 761 8857-224; http://www.ipm.fraunhofer.de 


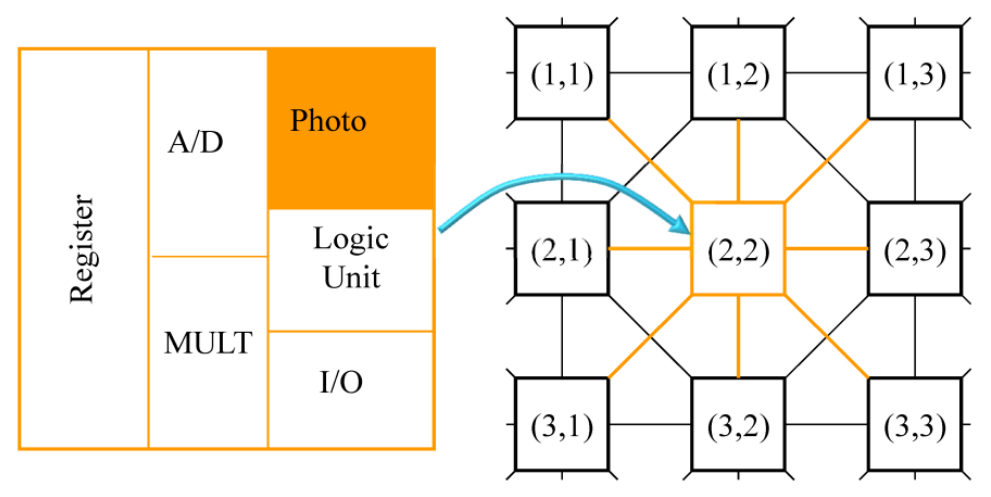

Figure 1. CNN enable the integration of a SIMD processor architecture into the electronic circuitry of CMOS camera pixels. Every cell is interconnected with its eight nearest neighbors ( $3 \times 3$ neighborhood).

\section{CELLULAR NEURAL NETWORKS (CNN)}

Cellular neural networks were originally introduced by Chua and Yang [11] as a regular array of locally interconnected analog processing elements called cells which operate in parallel. The dynamic behavior is determined by the cell connectivity pattern (spatial neighborhood) and a set of configurable parameters. Such networks can be implemented using the voltage in condensers as a state variable. Together with some memory elements, such systems form the so called "CNN universal machine" which is a universal computer in the sense of Touring [12]. The major advantage for the integration into the sensors of CMOS cameras is that it can be implemented in a small space with the aid of mixed signal CMOS processes in an array of optical sensors together with analogue and digital data processing elements [13] (figure 1). This is important for the sensitivity of the optical sensor, because the additional circuitry per pixel reduces the fill factor of the camera. For the Q-Eye chip which is used for the wire drawing application, the size of the cell is 33.6 $\mu \mathrm{m}$ and the size of the photo sensor is $7 \mu \mathrm{m}$, so the fill factor is about $4 \%$ [14]. The increased size of the cell also affects the resolution which typically lies in the range of $128 \times 128$ to $256 \times 256$ cells [13-18]. Although there are also FPGA based implementations [19,20], this paper concentrates on CMOS implemented "focal-plane sensor-processor chips" because these systems overcome the bottleneck of data transfer in real-time image processing.

The potential advantages of this technology are high computational speed, high frame rates, short latency times, and high computational power at low energy consumption. In contrast to other SIMD architectures like GPUs, CNN offer a 2D spatial neighborhood - most systems have a 3 × 3 cell connectivity pattern - and a temporal neighborhood. Therefore, they are very efficient for many low-level image processing operations like linear convolutions, morphological operations, thresholding or logical concatenation of binary images [21]. High computational speed combined with short data access times promise high frame rates and short latency times. For simple algorithms like thresholding combined with binary operations, frame rates of up to $100 \mathrm{kHz}$ for both, acquisition and image evaluation have been reported [17]. In combination with latency times in the range of $100 \mu \mathrm{s}, \mathrm{CNN}$ are in particular promising for closed-loop control of industrial processes $[22,23]$. Despite their computational speed, the typical energy consumption of such chips lies well below $1 \mathrm{~W}$ [14-16] which allows small form factors or mobile vision systems [24, 25].

However, CNN also have some disadvantages which are mainly due to their analogue nature. The computational accuracy is limited due to noise, spatial inhomogeneity between the cells, and fading, i. e. the loss of charges due to shunt currents in analogue memories [26]. In addition, divisions are restricted to a small number of integer values [21]. Trigonometric or arithmetic functions are not available in any of these platforms.

This shows that CNN-based cameras have specific properties which are not equally suited for every application. The high frame rate and the short latency times have the potential to enable new machine vision applications in surface inspection. Some previous publications investigate the system design for the inspection of metal properties [4, 27]. The aim of this paper is to proof that the computational properties are sufficient for industrial requirements. The system discussed hereafter is based on the Q-Eye [14], which consists of 176 x 144 cells with a 3 x 3 neighborhood. The Q-Eye is part of the Eye-RIS camera which contains an additional FPGA based NIOS II processor by Altera to control the operation of the whole vision system and to analyze the information output of the Q-Eye, e.g. performing all the decision-making and synchronization tasks. 


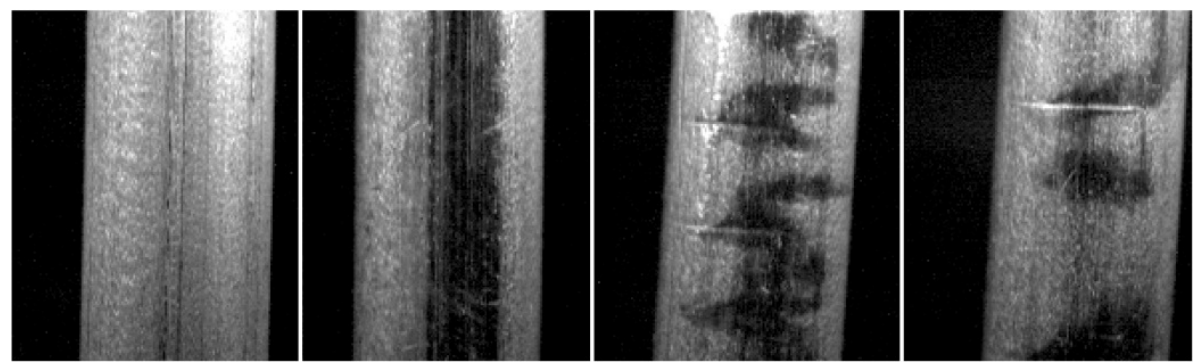

Figure 2. Surface defects of the Aluminum wire drawing process. From left to right: Small die marks, large die marks, chatter marks, and cross dents. Wire diameter is $3.3 \mathrm{~mm}$.

\section{SURFACE INSPECTION SYSTEM}

Wire drawing is a metal working process used to reduce the cross section of a wire by pulling it through a series of drawing dies. The profile of each die is designed to introduce a predefined series of strains which alter the microstructure of the wire material. Therefore, besides a plastic deformation certain material properties such as hardening or surface quality are achieved [28-30]. As a result of numerical simulations of the drawing process [4], four relevant defect classes were identified (figure 2). Small die marks are not yet critical for material quality, but they indicate some initial damage on the die surface. If they occur, the manufacturer is able to change the dies to avoid material degradation or waste on a large scale. Otherwise, the die surface deteriorates further and large die marks appear. Since wires are usually drawn through a series of dies, resonant vibrations occur. These vibrations cause chatter marks which are also critical for material quality. The severest impairments originate from cross dents perpendicular to the wire axis, because they induce shear forces shear forces which weaken or even break the wire. For the aluminum wires investigated in this paper, even small dents with a width of $100 \mu \mathrm{m}$ are critical for crack formation. This raises the need for surface inspection systems which are able to detect such defects at typical feeding rates of $10 \mathrm{~m} / \mathrm{s}$. As pointed out in the introduction, this application is not feasible for conventional line cameras because they do not reach the minimum frame rate of $200 \mathrm{kHz}$ required by the sampling theorem.

\subsection{Measurement setup for gapless surface inspection}

The aim is a measurement setup which enables a $100 \%$ surface inspection of cylindrical wires. Figure 3 a) sketches the measurement system consisting of four cameras and four illumination units where each has to cover a $90^{\circ}$ section of the wire. To show the feasibility, a combination of a single camera and two lightings for a $90^{\circ}$ section of the wire is sufficient (figure $3 \mathrm{~b}$ ). Due to symmetry the angle $\alpha$ between the lightings and the optical axis in the radial plane is 45 degrees. The tilt angle $\beta$ of the lightings towards the wire axis is introduced in order to avoid specular reflections towards the camera optics. For cylindrical wires with a diameter of $3.3 \mathrm{~mm}$ each camera has to observe a section with a width of $2.3 \mathrm{~mm}$ in y-direction and a depth of $0.5 \mathrm{~mm}$ in z-direction (figure $3 \mathrm{c}$ ). To that area, the lateral movements of the wire $( \pm 0.75 \mathrm{~mm})$ must be added. Therefore, the minimum width $B_{y}$ of the field of view is $3.8 \mathrm{~mm}$ and the depth $\Delta z$ of the measurement volume becomes $2 \mathrm{~mm}$. The height $B_{x}$ of the field of view is determined below.
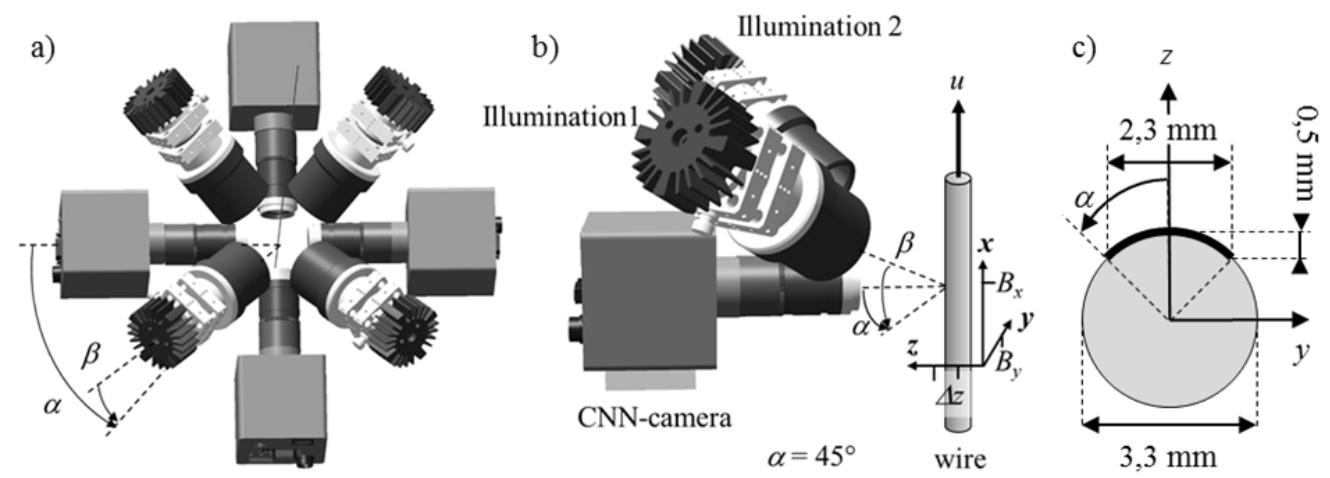

Figure 3. a) Measurement setup with four cameras and illumination units. b) Coordinate system for a single camera. c) $90^{\circ}$ section of a wire observed by a single camera mounted in z-direction. 

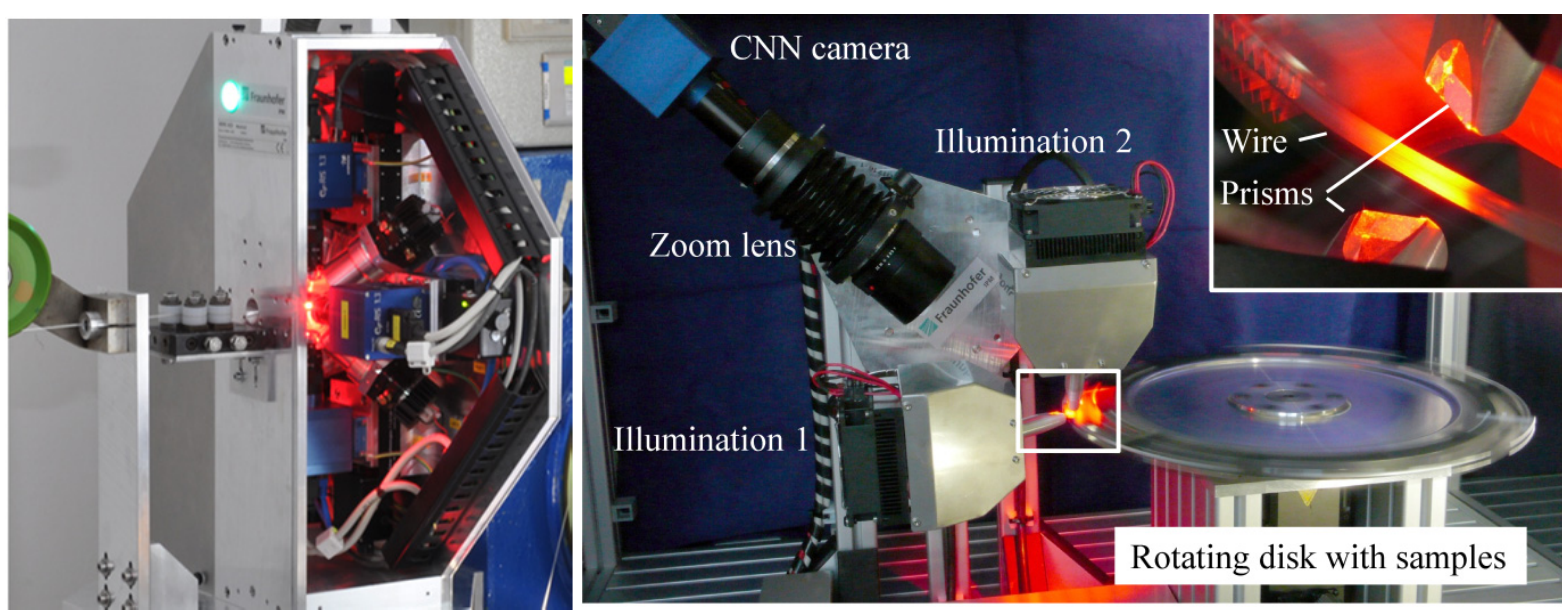

Figure 4. Left side: Measurement head with four cameras and illumination systems (diameter: $53 \mathrm{~cm}$ ). The wire runs from left to right. Right side: Test setup for measuring short samples with one camera and two illumination units (see figure $3 \mathrm{~b}$ ).

Figure 4 shows the final measurement head which is installed in a production line. Cameras and illumination units are arranged like in figure 3a). For the proof of feasibility, a test site with a single CNN camera and two illumination units was used. This test site has the advantage that short sample pieces with defects can be mounted on a rotating disk. Therefore, algorithms can be compared on a known set of samples and under well-defined conditions.

\subsection{Measurement requirements}

Within the measurement volume $B_{x} \cdot B_{y} \cdot \Delta z$, the optical resolution defined by the focal diameter $\Delta d$ is determined by the minimum defect size $\Delta l_{\text {min }}$. According to the sampling theorem the maximum focal diameter $\Delta d_{\max }$ of the camera optics must fulfill the condition

$$
\Delta d \leq \frac{\Delta l_{\min }}{2}
$$

A similar condition can be derived for the magnification $\gamma$ of the camera optics considering a defect of size $\Delta l_{\min }$ which is observed under the angle $\alpha$. This defect must be resolved with at least 2 pixels resulting in the condition

$$
\gamma \geq 3 \frac{\Delta l_{C N N}}{\Delta l_{\min }}
$$

where $\Delta l_{C N N}$ is the pixel pitch of the CNN camera. The factor 3 is chosen instead of $2 / \cos (\alpha)$ taking the $3 \times 3$ neighborhood of the CNN into account. To comply with these conditions throughout the depth $\Delta z$ of the measurement volume, f-number 11 was necessary for the camera optics.

With a given magnification $\gamma$ and the resolution of the CNN sensor of $M=176$ rows and $N=144$ columns, the field of view $B_{x} \cdot B_{y}$ can be calculated as

$$
B_{x} \leq \frac{M}{3} \Delta l_{\min }, \quad B_{y} \leq \frac{N}{3} \Delta l_{\min } .
$$

The result is a field of view with a width $B_{y}$ of $4.8 \mathrm{~mm}$ and a height $B_{x}$ of $5.8 \mathrm{~mm}$. This height $B_{x}$ and the feeding rate $u$ define the minimum frame rate $f$ for a $100 \%$ surveillance

$$
f \geq 2 \frac{u}{B_{x}}
$$


This condition defines a minimum overlap of $50 \%$ between two consecutive images. The reason is that the detection of large area defects like chatter marks shall be independent of their position in the image. It limits the computation time available for the evaluation of each image. For the wire drawing process with $u=10 \mathrm{~m} / \mathrm{s}$ and $B_{x}=5.8 \mathrm{~mm}$, the minimum frame rate $f$ becomes $3.5 \mathrm{kHz}$.

The exposure time $t_{E}$ is not only limited by the frame rate but also by the motion blur $b=u t_{E}$. In order to obtain a sufficient contrast for small defects, this motion blur must be smaller than the minimum defect size $\Delta l_{\min }$ resulting in the condition for the maximum exposure time

$$
t_{E} \leq \frac{\Delta l_{\min }}{u}
$$

According to this condition, maximum exposure time is $10 \mu \mathrm{s}$ for the wire drawing process.

This last condition for short exposure times contradicts condition (1) for small focal diameters over a large depth of focus. This contradiction must be resolved by a high brightness lighting system. In addition, on uneven metallic surfaces specular reflections must be avoided in order to achieve a homogeneous intensity distribution on the "good" object surface and making a high contrast for those features to be observed. Therefore, the lighting is essential for the performance. The LED based partial dark field illumination system used in the setup was described in detail in [26]. In the meantime, the exposure time was reduced to $10 \mu \mathrm{s}$.

\subsection{CNN Algorithms}

Algorithm 1 for the detection of cross dents uses the horizontal Sobel operator for edge enhancement (figure 5). The result is a line shaped local maximum near to a local minimum. In order to increase the robustness, both, the maximum and the minimum are filtered by a high threshold and a low threshold, respectively. Since the Sobel filter is a high pass filter, this operation is sensitive to noise. Therefore, both threshold images are combined by a pixel wise OR operation. The noise is filtered by a single dilation which closes the contour of the minimum and the maximum and a double erosion to delete the noise. Both, the Sobel and the morphological dilation/erosion operators exploit the spatial neighborhood of the CNN. Therefore, the runtime of this algorithm is about $40 \mu \mathrm{s}$.

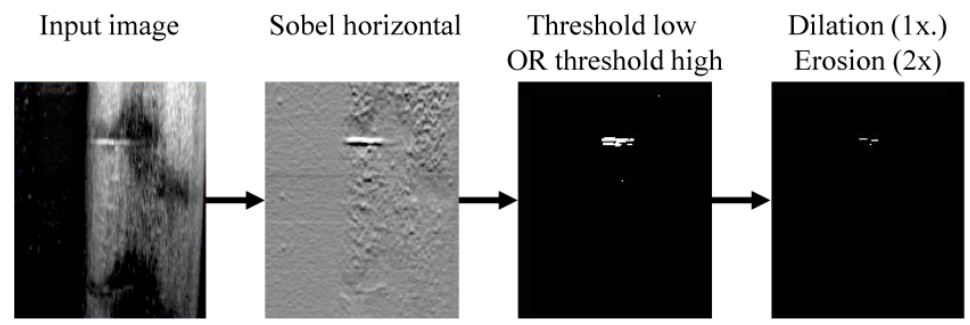

Figure 5. Algorithm 1: Detection of cross dents based on the horizontal Sobel operator (runtime $t_{\text {Algl }}=40 \mu \mathrm{s}$ ).

Algorithm 2 sketched in figure 6 is designed to detect large die marks and chatter marks. It exploits the vertical gradient introduced in the illumination by the angle $\beta$. In a combination with a global threshold, this leads to a variable size of the wire when dark spots appear on the wire surface. Since a dark field illumination is used, the brightness of the wire surface is defined by surface roughness. "Good" areas appear bright because they are rough. Die marks and chatter marks appear as dark spots because they have an increased specular reflectivity. Therefore, threshold values exist which are below the darkest grey values of "good" areas but which vary the bottom border of the wire within the threshold images in the presence of defects. These variations can be measured by executing a XOR-operation between consecutive threshold images. The size of the white areas is discriminated by five consecutive dilations and seventeen erosions. Both, die marks and chatter marks can be discriminated in time domain. Due to its irregular shape, chatter marks cause an alternating defect signal if three or more consecutive images are evaluated. This algorithm benefits from the temporal neighborhood between consecutive images. The runtime of this algorithm is $61 \mu \mathrm{s}$. This algorithm requires an overlap of about $50 \%$ between consecutive images to make it insensitive against defect position. 


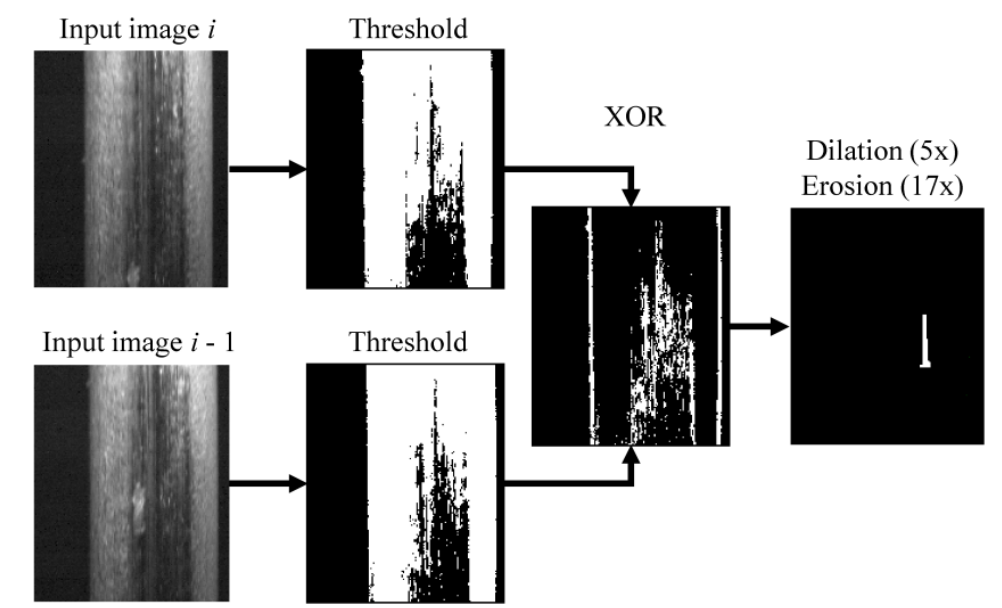

Figure 6. Algorithm 2: Detection of large die marks and chatter marks (runtime $t_{A l g 2}=61 \mu \mathrm{s}$ ).

Algorithm 3 for the detection of small die marks is the most complex one because it has to discriminate them from allowed structures with a similar size. The scheme is sketched in figure 7 . In order to limit the minimum defect size, the input image is smoothed by a so-called "diffusion" filter which averages the intensities of neighbored pixels. Since the die marks run in vertical direction, this filter is applied two times in horizontal and ten times in vertical direction. Afterwards, a vertical Sobel operator is applied for edge enhancement. Like in algorithm 1, the dark areas are filtered by a low and the bright areas by a high threshold value. Because the vertical Sobel filter is a first order derivative in horizontal direction, the dark vertical lines of the small die marks form neighbored negative edges on their left and positive edges on their right side. To discriminate the die marks from other structures, the negative edges on the left are shifted to the right and concatenated with the positive edges by the AND-operator. Again, noise is removed by some dilation and erosion operations.

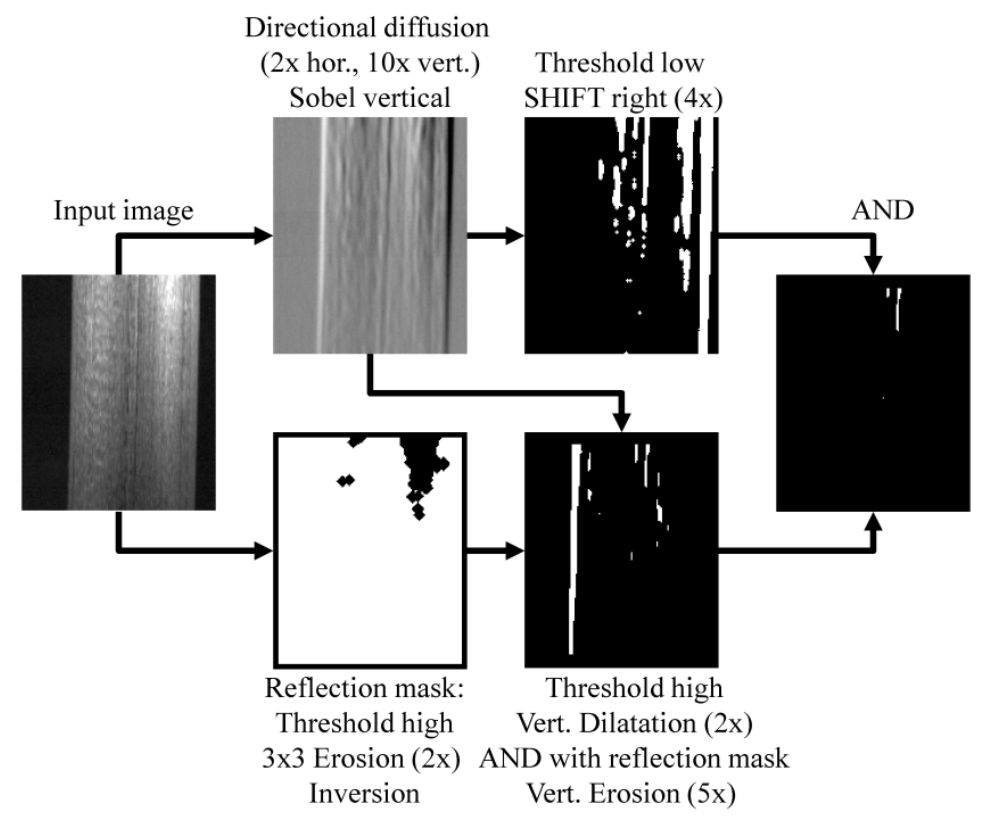

Figure 7. Algorithm 3: Detection of small die marks (runtime $t_{\text {Alg3 }}=115 \mu \mathrm{s}$ ).

All three algorithms return 'true' if white pixels remain in the result image and false otherwise. In order to optimize the frame rate of the CNN camera, the algorithms were aligned as shown in figure 8. Since algorithm 2 for the detection of the die marks and chatter marks requires two consecutive images, the shutter for image $i$ is opened while algorithm 1 evaluates image $i-1$. Afterwards, algorithms 2 and 3 are applied to images $i$ and $i-1$. The shutter time corresponds to the runtime of algorithm 2. Since the trigger must be set by the conventional NIOS processor, the exposure time is set by a 
current pulse of length $t_{E}$ which is delayed by a time $t_{D}$ (about $20 \mu \mathrm{s}$ ). The results of the algorithms are evaluated by the NIOS processor during the acquisition of image $i+1$. The duration $\tau_{P}$ of one period was about $244 \mu$ s resulting in a frame rate $f$ of roughly $4.1 \mathrm{kHz}$ when all algorithms are applied. Since the evaluation time of the results of the algorithms on the NIOS processor is negligible, the latency time is two times $\tau_{P}$ or $488 \mu \mathrm{s}$. When only algorithm 1 is applied for the detection of cross dents, the frame rate is limited by the illumination system to $10 \mathrm{kHz}$ and the latency time becomes $200 \mu \mathrm{s}$.

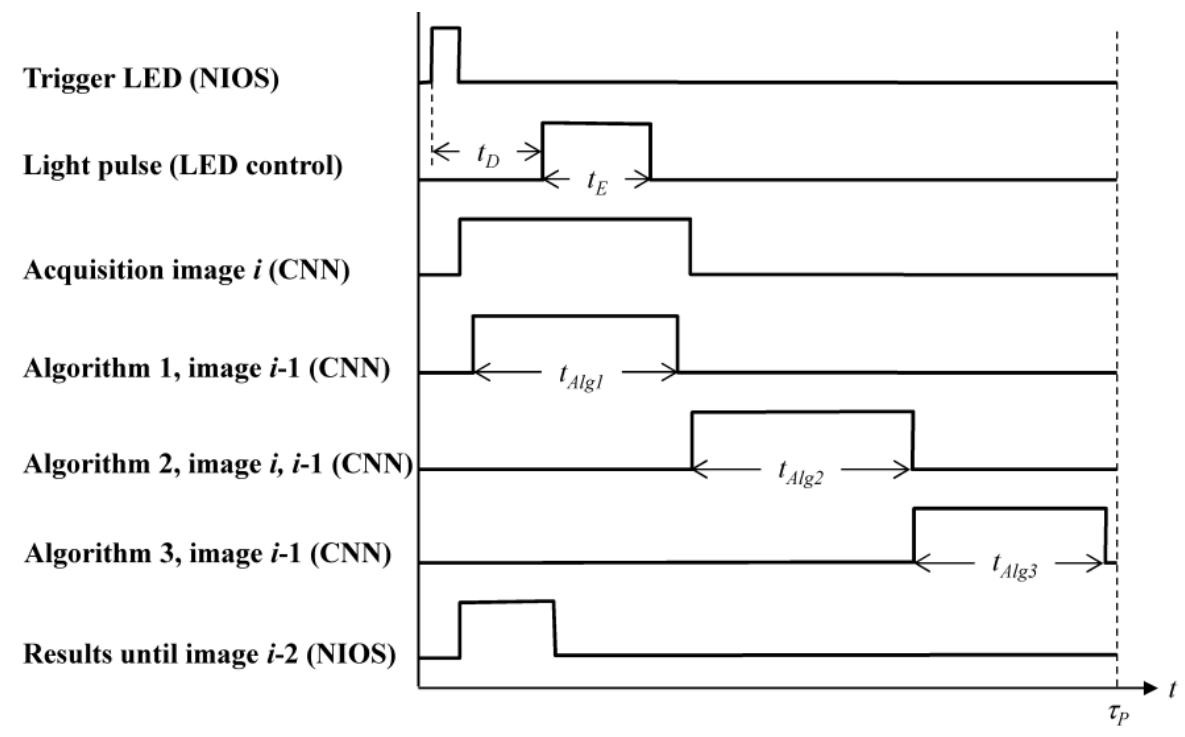

Figure 8: Temporal alignment of trigger, shutter, and algorithms.

\subsection{Demonstration of feasibility}

To demonstrate the feasibility of the wire drawing application, the requirements defined in section 3.2 have to be verified. Since conditions (1) to (3) are fulfilled due to the design of the optical system, the remaining task is the verification of conditions (4) and (5). The aim is to show that the CNN algorithms are sufficient for a safe detection of the defects shown in figure 2 in the presence of motion.
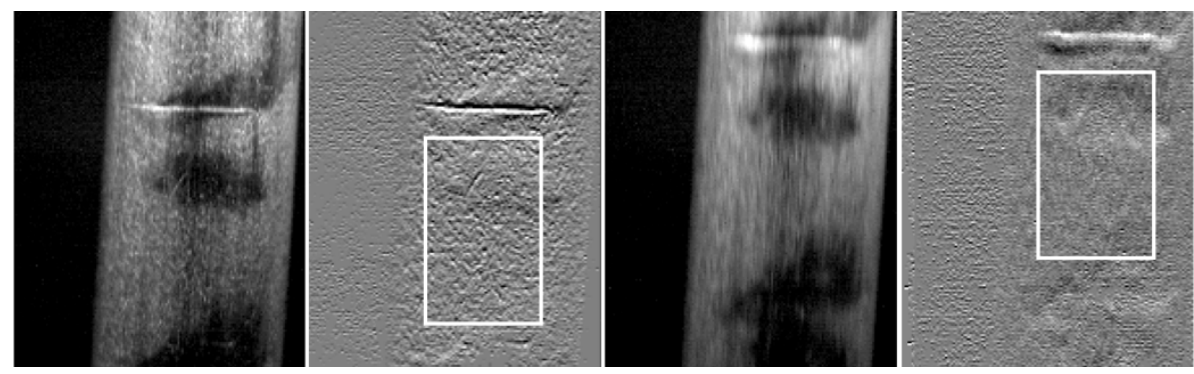

Figure 9: Influence of motion blur on a cross dent at feeding rates of 0 and $10 \mathrm{~m} / \mathrm{s}$. Left side: Camera images. Right side:

Normalized Sobel images. The standard deviation of the grey values in the rectangles is 33.5 and 30.7 digits.

The first task is to show that the optical resolution is sufficient in the presence of motion blur. Figure 9 shows measurement results of the most sensitive defect type: a cross dent with a vertical extend in the range of $100 \mu \mathrm{m}$. It shows two input images for algorithm 1 from the same cross dent acquired on the test site at 0 and $10 \mathrm{~m} / \mathrm{s}$ with an exposure time of $10 \mu \mathrm{s}$. The other two images are the corresponding images after the application of the horizontal Sobel filters which are the first derivative of the grey value in vertical direction. Since the cross dent appears as a bright line, a pair of a local minimum and maximum appears on the rising and the falling edge of the cross dent. To show the signal to noise ratio between the defect area and other parts of the wire surface, the intensity of the minimum is normalized to the grey value zero and the maximum to 255 . Afterwards the standard deviation of the grey values in the green rectangles was calculated as a measure for the noise. These noise values were 33.5 and 30.7 digits for feeding rates of 0 and $10 \mathrm{~m} / \mathrm{s}$. Therefore, the detectability of the cross dents in the Sobel image should not decrease at $10 \mathrm{~m} / \mathrm{s}$ despite the presence of motion blur. 
The second task is to show that the numerical accuracy of the $\mathrm{CNN}$ is sufficient for a safe detection and discrimination of the defect types shown in figure 2. To do this, a series of 501 reference images was used to test algorithm results (see table 1). Nearly half of the images are from good wire surfaces without any defect. These images were selected to represent the full range of "good" images during production as much as possible in order to make the algorithms robust against "false positive" detection, i.e. against defect detections on surfaces without defects. Nevertheless, the fraction of good images is much larger during production. Therefore, table one lists the detection rates of the algorithms from the previous section measured under the condition that no false detection occurs within the samples without defects. For cross dents, chatter marks, and large die marks, the detection rate under that constraint is $100 \%$. This high detection rate is in particular important for cross dents, because they appear only in one single image. All other defects extend over a number of images. Therefore, the detection rate for the small die marks is still sufficient.

Table 1: Detection rates measured on a series of reference images and frame rates for the CNN algorithms. For a safe detection of cross dents the frame is $10 \mathrm{kHz}$, for a safe detection and discrimination of all defect types is $4.1 \mathrm{kHz}$.

$\left.\begin{array}{|lrrr|}\hline \text { Defect type } & \text { Number of images } & \text { Detection rate } & \text { Frame rate } \\ \hline \text { No defect } & 226 & 0 \% \\ \text { Cross dents } & 10 & 100 \% \\ \text { Chatter marks } & 45 & 100 \% & \\ \text { Large die marks } & 91 & 100 \% \\ \text { Small die marks } & 129 & 97 \% & \end{array}\right] 4.1 \mathrm{kHz}$

So the results from table 1 show that the numerical accuracy is sufficient for robust defect detection in this application. For a practical application, defect discrimination is also desirable. This can be done by using subsequent images. For example both, chatter marks and large die marks are detected by algorithm 2 . To discriminate them from each other, the results of subsequent images can be taken into account. Whereas large die marks cause a continuous defect signal from algorithm 2 over several meters, chatter marks create an alternating signal. Among the chatter marks in our reference series, large die marks could be identified when the defect signal from algorithm 2 extends over more than three images.

This shows that both, defect detection and discrimination is possible with a frame rate of $4.1 \mathrm{kHz}$ which allows for gapless surface inspection.

\section{COMPARISON WITH CONVENTIONAL VISION SYSTEMS}

Due to the symmetry of the application, line cameras are preferred in conventional image processing systems. Since $\mathrm{CNN}$ have area image sensors, a figure of merit is needed for comparison. As such, the effective number of lines is used because it is directly linked to the minimum defect size $\Delta l_{\min }$. Due to the overlap of $50 \%$ between two consecutive images required by algorithm 2 , a line camera has to sample $M / 2$ lines for every image of the CNN camera - where $M=176$ is the number of rows in the $\mathrm{CNN}$ image. Therefore an effective line frequency $f_{L}$ can be defined for the $\mathrm{CNN}$

$$
f_{L} \equiv f \frac{M}{2}
$$

This means that the temporal resolution achieved with the CNN system at frame rates $f$ of $4.1 \mathrm{kHz}$ and $10 \mathrm{kHz}$ corresponds to the resolution of line camera systems with frame rates $f_{L}$ of $360 \mathrm{kHz}$ and $880 \mathrm{kHz}$, respectively. In the CNN system, the frame rate is limited by the computation time. Nevertheless, it is still above the maximum frame rates which current line cameras achieve due to data transport. Therefore, these results show that CNN cameras increase the temporal resolution of image processing systems.

Even if the camera interfaces become faster, an implementation of the CNN algorithms from section on conventional processors will be difficult. This is shown in table 2, which lists runtimes measured for some important image processing operators on a CNN chip [13] and on an Intel Core i7 processor [30]. The image size is the one of the CNN (176 x 144 pixels) and the code for the Core $\mathrm{i} 7$ was written in $\mathrm{C}++$ as a single threaded application using the Microsoft Visual Studio with optimization "fast" for a 64 bit platform. The binary images were also encoded as one bit per pixel. Therefore, the threshold operator, where this encoding is done, is quite slow and the AND operator benefits most. 
Table 2. Runtimes for image processing operators on 176 x 144 pixel images.

\begin{tabular}{|lccc|}
\hline Operator & Q-Eye & Core i7 & ratio \\
\hline Laplace & $27.3 \mu \mathrm{s}$ & $89 \mu \mathrm{s}$ & 3.2 \\
Sobel & $17.0 \mu \mathrm{s}$ & $91 \mu \mathrm{s}$ & 5.3 \\
threshold & $3.0 \mu \mathrm{s}$ & $184 \mu \mathrm{s}$ & 61.3 \\
AND between binary images & $2.4 \mu \mathrm{s}$ & $0.5 \mu \mathrm{s}$ & 0.2 \\
Erosion (1 iteration) & $4.1 \mu \mathrm{s}$ & $4.7 \mu \mathrm{s}$ & 1.1 \\
\hline Peak power consumption & $1 \mathrm{~W}$ & $45 \mathrm{~W}$ & 45 \\
\hline
\end{tabular}

Although the $\mathrm{C}++$ implementation uses just one of the four processor cores, the results show that the Intel processor cannot reach the frame rates of the Q-Eye. Even the simplest of the CNN algorithms (algorithm 1) consists of one application of the Sobel operator, two threshold applications, one OR concatenation, and three erosions (a dilation is the same as an erosion on the black pixels). Therefore this algorithm has a runtime of about $472 \mu$ s compared to $40 \mu$ s on the Q-Eye. This means that the Intel Core 17 will not reach a frame rate $4 \mathrm{kHz}$ with $\mathrm{CNN}$ resolution, even if the workload is distributed to all processor cores and if the computational efforts of data copying and thread synchronization are neglected.

Another advantage of the Q-Eye processor is the low power consumption. Regarding the measurement head on the left side of figure 4, the effort of cooling is reduced significantly. This simplifies the design of compact and sealed measurement systems enormously.

\section{CONCLUSION}

This article discusses new opportunities which CNN based cameras open for the inspection of metal surfaces as it is widely used in industrial quality control. The wire drawing process is used as an example application because there surface defects with a lateral size of $100 \mu \mathrm{m}$ have to be detected at a feeding rate of $10 \mathrm{~m} / \mathrm{s}$. This application is not feasible for state-of-the-art line cameras because they do not reach the minimum frame rate of $200 \mathrm{kHz}$ required by the sampling theorem. In addition, the runtime of the algorithms on an Intel Core i7 processor should be insufficient.

As an alternative, CNN based cameras were used because this technology enables the integration of SIMD processors into CMOS camera chips which eliminates the bottleneck of data transport. The reason is that CNN are implemented analogue devices which require a smaller area than digital processors. In comparison to GPUs, CNN offer a 2D spatial neighborhood and a temporal neighborhood. The spatial neighborhood accelerates image processing operators based on linear convolutions like Sobel filters or morphological operators like erosions. The temporal neighborhood is exploited by logical concatenations of binary images. Therefore, $\mathrm{CNN}$ are very efficient for many image processing applications in terms of computational speed, power consumption and latency.

Despite these advantages, $\mathrm{CNN}$ are not suitable for every application. Compared to cameras without focal plane processors, the integration of the SIMD architecture increases pixel size which reduces resolution (Q-Eye: $176 \mathrm{x} 144$ pixels) and fill factor (Q-Eye: $4 \%$ ) of the image sensor. In addition, it is an analogue technology with a limited computational accuracy for grey value operations. The measurement results of the wire drawing application prove that the accuracy is sufficient for many industrial surface inspection applications and that is possible to design image processing systems which outperform conventional ones. Using the relation between lateral feature size and surface speed as a figure of merit, the CNN based system outperforms existing quality control systems for the wire drawing application by an order of magnitude.

These results show that $\mathrm{CNN}$ which are integrated in CMOS camera chips have gained the maturity for a broader range of industrial applications. It is in particular suitable for real-time applications where high frame rates, short latency times, and low power consumption are required and where their accuracy of grey value operations is sufficient. 


\section{ACKNOWLEDGEMENT}

This work was financed by the Baden-Württemberg Stiftung gGmbH within the project "FlexFormCNN - Flexible Steuerung von Umformprozessen mit Cellularen Neuronalen Netzen". The authors also express their gratitude to Bernhard Blug and Andreas Kailer from Fraunhofer-Institute for Mechanics of Materials IWM for the reference samples and the numerical simulations of the wire drawing process.

\section{REFERENCES}

[1] Butter, M., Leis, M., Sandtke, M., McLean, M., Lincoln, J., Wilson, A., "The Leverage Effect of Photonics Technologies: the European Perspective. Final Report, March 2011," Study prepared for the European Commission, DG Information Society and Media under reference SMART 2009/0066. TNO, Delft (2011). http://cordis.europa.eu/fp7/ict/photonics/docs/reports/photonicsleveragestudy en.pdf.

[2] Shinohara, T., Yoshida, K., "Deformation analysis of surface flaws in stainless steel wire drawing," Journal of Materials Processing Technology 162-163, pp. 579-584, (2005).

[3] Baek, H.M., Jin, Y.G., Hwang, S.K., Im, Yong-Taek; Son, Il-Heon; Lee, Duk-Lak "Numerical study on the evolution of surface defects in wire drawing," Journal of Materials Processing Technology 212 (4), pp. 776-785 (2012).

[4] Blug, A., Strohm, P., Carl, D., Höfler, H., Blug, B., Kailer, A., "On the Potential of Current CNN Cameras for Industrial Surface Inspection," Proc. IEEE $13^{\text {th }}$ International Workshop on Cellular Nanoscale Networks and their Applications (2012).

[5] Basler AG, An der Strusbek 60-62, 22926 Ahrensburg, Deutschland: Basler sprint. Line scan cameras. http://www.baslerweb.com (2012).

[6] Mikrotron GmbH, Landshuter Str 20-22 85716 Unterschleißheim (Hg.): EoSens ® 4CXP. High-Speed CMOS Camera. Revision L/I. http://www.mikrotron.de/pdf/mikrotron_eosens_4cxp_dsh.pdf.

[7] "SIMAC 63. Oberflächenqualitäts-Prüfsystem mit CCD-Kameras \& Bildverarbeitung," Zumbach Electronic AG, Postfach CH-2552 Orpund, Swiss (2010). http://www.zumbach.com.

[8] Esteban, B., Riba, J.-R., Baquero, G., Ferrater, C., “An eddy-current-based sensor for preventing knots in metallic wire drawing processes," Nondestructive Testing and Evaluation 26 (2), pp. 169-180 (2011).

[9] Kreutzbruck, M., Allweins, K., Strackbein, C., Bernau, H., "HIGH RESOLUTION EDDY-CURRENT WIRE TESTING BASED ON A GMR SENSOR-ARRAY”, AIP Conference Proceedings Volume 1096 (2009).

[10] Nieto, A., López Vilarino, D., Sáncheztor, V. B., "Towards the Optimal Hardware Architecture for Computer Vision," Fabio Solari (Ed.) [Machine vision. Applications and systems.] InTech, Rijeka, New York (2012).

[11] Chua, L.O; Yang, L., "Cellular neural networks: Theory,” IEEE Transactions on Circuits and Systems, 35 (10), pp. 1273-1290 (1988).

[12] Roska, T., Chua, L. O., “The CNN universal machine: an analogic array computer,” IEEE Transactions on Circuits and Systems II: Analog and Digital Signal Processing. (3), pp. 163-173 (1993).

[13] Rodríguez-Vázquez, A., Liñán-Cembrano, G., Carranza, L., Roca-Moreno, E., Carmona-Galán, R., JiménezGarrido, F. et al., "ACE16k: The Third Generation of Mixed-Signal SIMD-CNN ACE Chips Toward VSoCs," IEEE TRANSACTIONS ON CIRCUITS AND SYSTEMS-I: REGULAR PAPERS 51 (5), pp. 851-863 (2004).

[14] Rodríguez-Vázquez, A., Domínguez-Castro, R., Jiménez-Garrido, F., Morillas, S., García, A., Utrera, C. et al. “A CMOS Vision System On-Chip with Multi-Core, Cellular Sensory-Processing Front-End,” Baatar, C., Porod, W., Roska, T. (Ed.) [Cellular Nanoscale Sensory Wave Computing,] Springer, Boston (2010).

[15] Fernandez-Berni, J., Carmona-Galan, R., Carranza-Gonzalez, L., "FLIP-Q: A QCIF Resolution Focal-Plane Array for Low-Power Image Processing," IEEE J. Solid-State Circuits 46 (3), pp. 669-680 (2011).

[16] Carey, S. J., Barr, D. R. W., Dudek, P., "Demonstration of a low power image processing system using a SCAMP3 vision chip," Proc. IEEE $13^{\text {th }}$ International Workshop on Cellular Nanoscale Networks and their Applications (2012).

[17] Carey, S. J., Barr, D. R. W., Wang, B., Lopich, A., Dudek, P., "Locating High Speed Multiple Objects using a SCAMP-5 Vision-Chip," Proc. IEEE $13^{\text {th }}$ International Workshop on Cellular Nanoscale Networks and their Applications (2012).

[18] Zarándy, Ákos (Ed.) [Focal-Plane Sensor-Processor Chips,] Springer Science+Business Media LLC, New York (2011).

[19] Nagy, Z., Szolgay, P., "Configurable multi-layer CNN-UM emulator on FPGA,” IEEE Transactions on Circuits and Systems I: Fundamental Theory and Applications, (50), pp. 774-778 (2003). 
[20] Müller, J., Becker, R., Müller, J. ; Tetzlaff, R., “CESAR: Emulating Cellular Networks on FPGA,” Proc. IEEE 13 ${ }^{\text {th }}$ International Workshop on Cellular Nanoscale Networks and their Applications (2012).

[21] Zarándy, A., Rekeczky, C., "2D operators on topographic and non-topographic architectures-implementation, efficiency analysis, and architecture selection methodology,” Int. J. Circ. Theor. Appl. 39 (10), pp. 983-1005 (2011).

[22] Blug, A., Abt, F., Nicolosi, L., Heider, A., Weber, R., Carl, D. et al., "The full penetration hole as a stochastic process: controlling penetration depth in keyhole laser-welding processes,” Appl. Phys. B 108 (1), pp. 97-107 (2012).

[23] Strohm, P., Blug, A., Carl, D., Höfler, H., “Zellulare Neuronale Netzwerke zur schnellen bildbasierten Regelung von Materialbearbeitungsprozessen. Cellular neural networks for the fast and image based control of industrial joining and shaping processes," tm - Technisches Messen 79 (3), pp. 152-158 (2012).

[24] Pazienza, G.E., Ponce-García, X., Balsi, M., Vilasís-Cardona, X., "Robot vision with cellular neural networks: a practical implementation of new algorithms," Int. J. Circ. Theor. Appl 35 (4), pp. 449-462 (2007).

[25] Alba, L., Arena, P., Fiore, S. de; Patane, L., Strauss, R., Vagliasindi, G., "Implementation of a drosophila-inspired orientation model on the Eye-Ris platform," Proc. IEEE $12^{\text {th }}$ International Workshop on Cellular Nanoscale Networks and their Applications (2010).

[26] Misra, J., Saha, I., “Artificial neural networks in hardware: A survey of two decades of progress," Neurocomputing (74), pp. 239-255 (2010)

[27] Blug, A., Jetter, V., Strohm, P., Carl, D., Höfler, H, "High power LED lighting for CNN based image processing at frame rates of $10 \mathrm{kHz}$," Proc. IEEE $12^{\text {th }}$ International Workshop on Cellular Nanoscale Networks and Their Applications (2010).

[28] Lo, Sy-Wie; Lu, Yuung-Hwa, "Wire drawing dies with prescribed variations of strain rate," Journal of Materials Processing Technology 123, pp. 212-218 (2002).

[29] Segal, V.M., "Severe plastic deformation: simple shear versus pure shear," Mater Sci Eng. A 338, pp. 331-344 (2002).

[30] Phelippeau, A.; Pommier, S.; Tsakalakos, T.; Clavel, M.; Prioul, C., “Cold drawn steel wires—processing, residual stresses and ductility_part I: metallography and finite element analyses," Fatigue \& Fracture of Engineering Materials \& Structures 29 (3), pp. 243-253 (2006).

[31] Intel Core i7-2720QM, 2.2-3.3 GHz, 64 bit, SSE3 commands, 4 kernels, 8 threads, 32 nm structure size, peak power consumption $45 \mathrm{~W}$. http://www.intel.com 